\title{
LA SÉPTIMA GRAN COSMOVISIÓN: MECÁNICA CUÁNTICA Y UNIVERSO CONSCIENTE
}

\author{
ADOLFO CASTILLA \\ Universidad Antonio de Nebrija, Madrid
}

\begin{abstract}
RESUMEN: Se aborda en este trabajo un tema tan antiguo y general, pero al mismo tiempo tan actual, como las diferencias y posibles relaciones entre la materia y el espíritu. Se hace a través de la consideración de fenómenos como las "cosmovisiones", o interpretaciones generales de lo que es nuestro mundo y lo que los hombres hacemos en él, surgidos periódicamente en la humanidad como consecuencia de grandes descubrimientos y grandes teorías explicativas de la naturaleza de nuestro universo, incluyendo al hombre dentro de él. Sugerimos que estamos entrando en la séptima cosmovisión y la definimos en términos generales. El perfeccionamiento de la especie humana fisiológica y mentalmente puede que sea uno de los resultados concretos a los que pueden dar lugar las nuevas interpretaciones y los cambios tecnológicos que las acompañan, así como la utilización de la Inteligencia Artificial Fuerte y la creación de robots humanoides y «máquinas espirituales». Más importante que todo ello, y a lo que especialmente nos referimos, es el hecho de que la nueva etapa del hombre ante la que estamos, puede caracterizarse por un mayor papel del espíritu, la consciencia, y la subjetividad en la marcha de nuestro mundo, algo a lo que contribuyen las nuevas concepciones relacionadas con la Mecánica Cuántica y con la posibilidad de existencia de un Universo Consciente.
\end{abstract}

PALABRAS CLAVE: cosmovisión, espíritu, conciencia, subjetividad, inteligencia artificial fuerte, convergencia NBIC, transhumanismo, hombre posthumano

\section{The Seventh Great Cosmovision: Quantum Mechanics and Conscious Universe}

ABSTRACT: This paper deals with the very general and old theme, but at the same time a very current one, of differences and possible relationships between matter and spirit. It is done through the consideration of phenomena so call, "worldviews", or general interpretations of what is our world and what men do in it, which emerge periodically in humanity as a result of great discoveries and great explanatory theories on the nature of our universe, including man within him. It is suggested that we are entering the seventh "worldview» in the history of humankind and it is defined in general terms. The enhancement of humans in physiological and mental terms may be one of the concrete results of the new interpretations and technological changes that accompany them, as well as the pervasive use of Strong Artificial Intelligence, creation of humanoid robots and the construction of «spiritual Machines». More important than all this, and to what we refer especially, is the fact that the new stage of man before him, can be characterized by an increased role of spirit, consciousness, and subjectivity in the progress of our world, something to what new ideas related to Quantum Mechanics and the possibility of existence of a Conscious Universe contribute in an important manner.

KEY WORDS: worldview, spirit, consciousness, subjectivity, strong al, NBIC convergence, transhumanism, posthuman

Imperfecta es la vida de los hombres en este planeta, como seguramente se ha escrito miles de veces antes de ahora. Imperfecta e inaceptable para un alto porcentaje de la humanidad, a pesar de las aportaciones sin fin del hombre al mundo en el que habita y de algo a lo que llamamos progreso. La supervivencia es difícil para muchos miembros de nuestra especie, pero para muchos más es imposible el acceso a la educación, a la cultura, al arte, al conocimiento y al pensamiento elaborado y la racionalidad, dimensiones de la experiencia humana que muchos consideramos dan sentido a nuestra existencia y la justifican. 
Es preocupante en esas condiciones que unos nos dediquemos a reflexionar mientras otros intentan sobrevivir. Sobre todo cuando la mente del hombre, esa evanescente capacidad de generar ideas abstractas de la que parece haber surgido todo lo que existe a su alrededor, ha sido incapaz hasta ahora de crear un mundo razonablemente satisfactorio para todos. Sublime a veces en lo que genera en términos de ideas, teorías, explicaciones, belleza y mundo físico, la mente puede ser también fuente de ideologías falsas, errores, fealdad y materialidad perniciosa.

Lo más curioso es que casi todo lo anterior y casi todo lo que vemos en el mundo construido por el hombre comienza por la aparición de una idea abstracta en su mente la cual termina transformando la realidad material en la que vive. Para bien o para mal eso muestra que hoy, después de muchos años de evolución y sin entrar de momento en el tema de qué da pie a qué - lo físico a lo abstracto o lo abstracto a lo físico somos mezcla de materia y subjetividad, de naturaleza física y naturaleza mental o espiritual. Las dos son realidades de nuestras vidas que debemos potenciar y poner a disposición de hacer un mundo más viable y un universo más habitable. Con la particularidad de que llevamos mucho tiempo dando prioridad a la primera de ellas y olvidándonos de la segunda.

No sabemos por qué pero muchos científicos y muchos filósofos, de hecho, llevan tiempo queriendo marginar o eliminar de lo que somos a la subjetividad y a la consciencia, esperando quizá que la ciencia explique pronto cómo los «qualia», es decir, las cualidades subjetivas de las experiencias individuales, surgen del mundo físico y material de las neuronas, las sinapsis y los impulsos eléctricos que se producen del diferencial de potencial a ambos lados de las membranas de dichas células cerebrales.

No parece fácil sin embargo que tal explicación se produzca ni a corto ni a largo plazo. Y más bien puede que lo que necesitemos hoy sea reconocer la existencia de esos dos componentes de la naturaleza humana, o dos naturalezas si lo expresamos de otra manera, y avanzar en paralelo en ellos sabiendo que los dos son complementarios en el hombre. El pensamiento, la subjetividad y la consciencia en general, constituyentes de la segunda naturaleza mencionada, y común a todos los hombres, es lo que parece que nos toca llevar ahora a niveles de evolución superiores a los conseguidos. No en vano estamos en la sociedad de la información y del conocimiento y no en vano nos adentramos como nunca hasta ahora en el interior de nuestra naturaleza física y nuestra fisiología, con particular referencia al cerebro y a la mente, así como en las profundidades y lejanías de nuestro universo ${ }^{1}$.

En este breve trabajo pretendo, en línea con lo que se hace hoy cada vez con más intensidad, hablar de los componentes básicos de la naturaleza del hombre: materia y espíritu. Son los situados en primer lugar, pero no debemos olvidar otros componentes de dicha naturaleza, a los que me referiré en otra ocasión, como la energía, la información, la comunicación y, muy especialmente, el propósito, la intencionalidad y el destino.

\section{Una NUEVA ETAPA ANTE NOSOTROS DE GRANDES AVANCES CONCEPTUALES}

Para muchos pensadores, intelectuales y científicos actuales nos encontramos a las puertas de grandes descubrimientos y grandes avances conceptuales y ello significa que nuestras interpretaciones sobre el mundo físico en el que habitamos pueden cambiar

\footnotetext{
1 No es el dualismo cartesiano al que hacemos referencia, es, en todo caso, un «dualismo propietario», o dicho de otra manera, que lo físico y lo subjetivo son las dos caras de una misma moneda, que en este caso es el hombre.
} 
de forma sustancial y la vida de los hombres verse afectada considerablemente. Lo dice una vez más Lisa Randall, catedrática de física el Universidad de Harvard, refiriéndose a los avances en la física de partículas y en la cosmología en su más reciente publicación «Llamando a las puertas del cielo» (Randall, 2013) ${ }^{2}$

El razonamiento, la lógica, la argumentación, la racionalidad y en definitiva el intento de acercarnos a la verdad, debe ser, como siempre, el instrumento básico para dar a luz a ese mundo nuevo que es posible. Pero, para ello, necesitamos más que nunca un pensamiento comprensivo, sistémico, global, holístico. Un pensamiento que no sea sólo especialización y conocimiento de cada más de cada vez menos, repetición de lo que otros han dicho, erudición sin fundamento y mera reflexión intelectual sin conexión al mundo físico y material que nos constituye y a la realidad tecnológica que nos circunda.

Un pensamiento también que sepa sacar lo mejor del pensamiento postmoderno, irracional, débil y deconstructivista de los últimos años, que como muchas otras aportaciones conceptuales del hombre debe ser tenido en cuenta.

Y, desde luego, no un pensamiento cerrado, único y sistémico en el mal sentido de este concepto, a la imagen de lo peor del idealismo alemán racionalista de los siglos XVIII y xIX.

Un pensamiento, por último, no necesariamente, o exclusivamente, ligado a los pensadores profesionales ya sean filósofos, científicos o intelectuales sin más. Alguien, no alejado de esos mundos pero tampoco agostado por ellos, debe dedicarse a dar sentido total a nuestro mundo, como muy bien sugirió Erwin Schrödinger en su famoso libro ¿Qué es la vida? (Schrödinger, 2006)

Las reflexiones intelectuales en definitiva, como la presente, sólo están justificadas si aportan algo a la vida del hombre y al buen funcionamiento de sus sociedades. Con esa intención las llevamos a cabo y con ella ponemos énfasis en la posible aparición entre nosotros de una nueva gran «cosmovisión».

Por cosmovisión entendemos aquí una visión general del mundo distinta de la existente previamente que los hombres adquieren periódicamente por motivos diversos. Se refiere en general al entorno del hombre y al mundo natural en el que habita y está formada por determinadas percepciones, interpretaciones, conceptualizaciones y valoraciones. Puede relacionarse con la manida y odiosa expresión — por su excesivo y no siempre justificado uso-, de «cambio de paradigma», pero es mucho más general y comprensiva. La palabra se ha utilizado con frecuencia con sentido religioso, ya que bajo ella se acumulan opiniones y creencias sobre cómo son las cosas. En este trabajo se une mucho a las nuevas leyes sobre el funcionamiento de la naturaleza que el hombre va descubriendo.

Si no nos equivocamos mucho estamos a las puertas de una nueva interpretación general de lo que es el hombre y el universo en el que habita, la séptima según mis cálculos, desde los albores de la humanidad, la cual como todas las verdaderas cosmovisiones es fundamentalmente producto de descubrimientos científicos de importancia desveladores de dimensiones, fenómenos y leyes nuevas sobre la naturaleza de nuestro

2 «Estamos al borde del descubrimiento. Están en curso los mayores y más apasionantes experimentos en física de partículas y cosmología, y muchos de los mejores físicos están pendientes de sus explicaciones». Página 7

3 «Yo no veo otra escapatoria frente a ese dilema (de saber cada vez más de cada vez menos) (si queremos que nuestro verdadero objetivo no se pierda para siempre) que la de proponer que algunos de nosotros se aventuren a emprender una tarea sintetizadora de hechos y teorías, aunque a veces tengan de ellos un conocimiento incompleto e indirecto, y aun a riesgo de engañarnos a nosotros mismos». Páginas 13 y 14 . 
universo. Descubrimientos que afectan a las ideas y concepciones de lo que los hombres somos y hacemos en este mundo, y que terminarán afectando a la tecnología que desarrollaremos, a las economías que adoptaremos y a los estilos de vida que finalmente practicaremos. Nos referiremos más adelante a esta cuestión de las cosmovisiones, pero de momento queremos hacer tres afirmaciones previas: 1) que en nuestras reflexiones debemos tener en cuenta las cuatro habilidades básicas del hombre, la manual, artesanal o tecnológica; la artística; la intelectual; y la científica; 2) que vivimos un cambio tecnológico acelerado, especialmente en el terreno de las tecnologías de la información, la comunicación y el conocimiento; y 3) que la nueva cosmovisión en procesos de formación está basada en las concepciones de la mecánica cuántica, en la convergencia NBIC (nanotecnología-biotecnología-infotecnología-cognotecnología) y en los avances en cosmología, terrenos muy activos en la actualidad en los que, además, surgirán en breve nuevos y destacados descubrimientos.

\section{LAS CUATRO CAPACIDADES BÁSICAS DEL HOMBRE}

Hoy es muy común que los filósofos se acerquen a la ciencia y que los científicos, sobre todo los físicos, hagan filosofía. Todo el mundo parece estar de acuerdo en que ambas, filosofía y ciencia, son actividades intelectuales, aunque en la segunda tiene siempre las ventajas de referirse a la naturaleza y a los hechos objetivos y de disponer de la demostración para que su curso no se tuerza y sus avances se consoliden.

Puede que hoy haya más interés que nunca en que los filósofos y científicos se acerquen e, incluso, que los primeros quieran construir sus interpretaciones a partir de los descubrimientos de los segundos. Así ocurre, por ejemplo, con los llamados «neurofilósofos», pero debemos decir que los filósofos se han preocupado por el conocimiento de la naturaleza desde muy antiguo y que la "filosofía de la ciencia» ha existido más o menos desde siempre aunque no con ese nombre preciso. Esta denominación, de hecho, fue adoptada, o al menos difundida, por el Círculo de Viena a primeros del siglo xx. Miembros iniciales de esa escuela fueron, su fundador Moritz Schlick, Rudolf Carnap, Otto Nuerath, Charles Morris y otros.

Como es sabido, esta escuela de pensamiento preconizaba una concepción científica del mundo, defendía el empirismo y pretendía la unificación del lenguaje de la ciencia y la eliminación de la metafísica. Dio lugar a denominaciones como el positivismo lógico y el empirismo lógico o neo-empirismo y resultó con el tiempo demasiado estricta en sus planteamientos. Desapareció, de hecho, como escuela de pensamiento, en 1936, aunque en los Estados Unidos y en otros países siguió practicándose de una manera dispersa.

Confirmándose de esa manera que la ciencia y su método no pueden arrinconar al espíritu y a la subjetividad, ni tampoco imponerles su lógica o su forma de hacer las cosas.

La filosofía de la ciencia no desapareció, desde luego, con el Círculo de Viena y a lo largo del siglo xx ha habido grandes filósofos dedicados a esta materia como Karl Popper, Thomas Kuhn, Paul Feyerabend, Imre Lakatos, Ilya Prigogine y muchos otros. Todos ellos han estado ligados a la ciencia y a sus avances, y algunos, como Prigogine, han sido científicos destacados. Prigogine ganó el Premio Nobel de Química de 1977 por sus trabajos sobre el concepto de «estructuras disipativas» por él acuñado.

La filosofía analítica que se ha desarrollado a lo largo del siglo xx en los países anglosajones es en cierta forma una continuación del positivismo lógico aunque sin llegar a sus excesos. El enfoque de esa filosofía es la claridad y la lógica de las argumentaciones, el análisis del lenguaje y el respeto profundo a las ciencias naturales. 
Un filósofo español de la ciencia muy destacado, Jesús Mosterín (nacido en 1941), ha escrito importantes trabajos sobre la naturaleza humana, las relaciones filosofía-ciencia y la racionalidad actual, muy relacionados con los conocimientos científicos. En uno de sus últimos libros, Ciencia, filosofía y racionalidad, se refiere al continuo que forman y deben formar estas dos materias (Mosterín, 2013)4.

Yo no creo que sea exactamente como dice este autor pero sí creo que los descubrimientos científicos más radicales comienzan con puras especulaciones intelectuales y que después de que se descubra y se demuestre algo importante es la reflexión, digamos que filosófica, la que determina su valor, su capacidad de impacto en la vida de las personas y su significado para los hombres y sus sociedades.

Reconociendo la profesionalidad, el nivel filosófico-científico, y la altura intelectual de Mosterín, del que me considero seguidor y seguramente «alumno invisible», debo decir que hay tres aspectos de lo que se indica en su libro con los que no coincido totalmente.

El primero tiene que ver con ese continuo entre filosofía y ciencia del que habla. Sobre ello pienso que el filósofo puede tratar de explicar todo lo existente en nuestro mundo pero necesitamos distinguir entre, «hablar de algo», "hacer algo» $\mathrm{y}$ "ser algo».

Para lo primero los filósofos, son sin duda los más adecuados, pero no exactamente para los dos segundos. En el arte se ve todavía más claro que en la ciencia. Un filósofo especialista en estética puede explicar muy bien lo que hay detrás de un cuadro, lo que representa, lo que refleja de la mente del autor y muchas cosas más, pero difícilmente podrá él pintarlo, y aunque termine haciéndolo, difícilmente podrá «ser» un pintor.

En una palabra, la filosofía y la ciencia forman quizá un continuo en cuanto a hablar y explicar pero no, ni mucho menos, en cuanto a «hacer ciencia» y en cuanto a "ser un científico». Mucho más acusadas pueden ser las diferencias en cuanto a la tecnología y en cuanto al arte

No creo, en consecuencia, que exista un continuo entre la reflexión y las otras capacidades del hombre y esto para mi es importante porque incide en algo en lo que creo: la existencia en algún momento de dos mundos, o dos naturalezas, la material y la espiritual, y una vez admitida esa diferencia, aunque sea "propietaria», como dice John Searle, saber que las dos se dan en el mismo hombre y son parte de su «humanidad» que es única.

Con un corolario importante relativo a la necesidad que tenemos de unir esas dos dimensiones sin dejar que una acorrale o elimine a la otra. Algo a lo que parece llevarnos las interpretaciones de la Mecánica Cuántica, aunque sea muy prematuro hablar aquí de ello.

El segundo tema en el que no coincido con Mosterín es en la necesidad que tenemos los hombres de pensar, no sólo sobre la ciencia sino sobre el arte, la tecnología y, por supuesto, sobre la misma filosofía. Los hombres, como hemos adelantado, tenemos cuatro habilidades básicas fundamentales, una primera, la manual, artesanal, técnica o tecnológica; una segunda, la estética o artística; una tercera, la intelectual; y una cuarta, la científica. Hablar del hombre no es sólo referirse a lo que piensa y a lo que investiga, sino a lo que hace con sus manos y a lo que expresa en sus manifestaciones artísticas.

\footnotetext{
4 «Ciencia y filosofía forman un continuo. La filosofía es la parte más global y reflexiva del continuo, es escenario de las discusiones que preceden y siguen a los avances científicos. La ciencia es la parte más especializada, rigurosa y bien contrastada, la que se incorpora a los modelos estándar, a los libros de texto y a las aplicaciones tecnológicas. Ciencia y filosofía se desarrollan dinámicamente, en constante interacción. Lo que ayer era especulación filosófica hoy es ciencia establecida». Página 22
} 
En términos históricos la más antigua es la primera ya que surge con el homo faber y desde luego con el homo habilis, puede que hace más de 2 millones de años, en el Paleolítico Inferior. Es una habilidad consistente en construir con sus manos herramientas, utensilios, aparatos y máquinas potenciadores de sus capacidades físicas. Habilidad que le ha permitido sobrevivir, adaptarse a su mundo natural y con el tiempo transformarlo y explotarlo.

Es la habilidad o capacidad más antigua del hombre y la que sigue siendo un componente principal de lo que es y de lo que hace en este mundo. No hay duda a este respecto de que el hombre es un animal técnico, o tecnológico, y de que la tecnología ha sido hasta ahora un componente fundamental de la evolución de la humanidad. El lenguaje mismo es considerado por muchos como una de las primeras tecnologías humanas y lo mismo se puede decir, y con mayor propiedad, de la escritura.

Detrás del ascenso del hombre hacia lo que es hoy está su cerebro y su mente, es decir, todo es inicialmente intelectual y todo nace en el hombre por la aparición de una idea abstracta, pero hay que reconocer que la actividad intelectual o reflexiva que se necesita para hacer tecnología, por ejemplo, es menor que para otras actividades.

Algo parecido se puede decir de la habilidad artística o estética del hombre, la segunda en desarrollarse en términos históricos. Las pinturas rupestres nos hablan de ella.

Los griegos, especialmente los presocráticos y en gran manera los de la Grecia Clásica, desarrollan la tercera gran capacidad del hombre, la de razonar, argumentar y utilizar la lógica y la retórica.

La cuarta, como se sabe, es producto de la Revolución Científica de los siglos xvi y XVII europeos. Hablando siempre desde una perspectiva occidental.

El tercer punto de no coincidencia tiene que ver con el término cosmovisión. El libro de Mosterín es importante también para este artículo porque se refiere en varias ocasiones a las cosmovisiones, aunque su autor no las interpreta de la misma forma que lo hago yo.

Habla por ejemplo de que los filósofos y científicos deberían definir la cosmovisión actual, algo en lo que yo no creo, debido a que mi interpretación de las cosmovisiones tiene que ver con las nuevas concepciones sobre nuestro mundo que emergen periódicamente de forma espontánea en las mentes de muchas personas más o menos a la vez, como consecuencia de grandes descubrimientos o grandes nuevas teorías sobre la naturaleza de nuestro universo.

Tampoco coincido en el uso de éste concepto con el que se supone que es su introductor en tiempos modernos, el filósofo alemán, Wilhelm Dilthey (1833-1911). Este gran filósofo utilizó, como sabemos, el término alemán, Weltanschauungen, traducido al inglés como Worldviews, y consideró en su época que habían existido tres básicas interpretaciones de nuestro mundo: el naturalismo, el idealismo de la libertad o idealismo subjetivo y el idealismo objetivo.

Yo creo que las cosmovisiones no están sólo relacionadas con las ideas sino, y muy especialmente, con las interpretaciones científicas sobre el mundo y la materia, con las tecnología y máquinas que el hombre desarrolla y con el arte que produce.

Me refiero a continuación a mi propia interpretación de este término el cual debe aunar las cuatro capacidades del hombre a las que hemos hecho referencia.

\section{COSMOVISIONES: UNA BREVE INTRODUCCIÓN}

En un trabajo reciente sobre la digitalización y la automatización de todo tipo de la información he hecho referencia a un artículo de Manuel Castells incluido en un 
informe sobre las TICs e Internet publicado por el BBVA con el nombre de «Cambio. Cómo Internet está cambiando nuestras vidas» ${ }^{5}$. Cita en él un trabajo de Martin Hilber publicado en Science en 2011 según el cual a finales de 2010 el 95 \% de toda la información disponible en el planeta estaba digitalizado y la mayor parte, accesible en Internet y otras redes informáticas ${ }^{6}$. A mi me parece un simplificación del tema de la información, ya que todo el universo está hecho de información y las posibilidades de digitalización se acercan al infinito. Ahora por ejemplo estamos entrando en el interior de la fisiología humana, en el interior de su cerebro y en los espacios siderales del universo. Todo en esos mundos es información y todo se podrá digitalizar con el tiempo.

En relación con esa cuestión de la información en nuestro universo y con los nuevos descubrimientos científicos y las nuevas cosmovisiones creadas por ellos, estimo útil mencionar, sólo para poder hacer un ejercicio de prospectiva final en términos de un escenario previsible, que estamos a las puertas de una nueva interpretación y concepción global de nuestro universo. Está en parte causada, como ya he adelantado, por los últimos impactos de la Teoría de la Relatividad y los más recientes de la Teoría Cuántica, las dos todavía en fase de difusión en la sociedad a pesar del gran «choque» mundial de la primera en los años 20 del siglo pasado, y de datarse las concepciones ortodoxas de la segunda, en los años 30 a 40, también del siglo pasado.

Vengo desde hace tiempo llamando "cosmovisiones» a esas nuevas interpretaciones, en gran manera científicas, sobre la naturaleza de nuestro universo y sobre las leyes, en principio físicas, que lo gobiernan y al nuevo mundo por ellas generado. Hasta ahora creo que se han producido siete grandes cosmovisiones de ese tipo y todas ellas han dado lugar a un nuevo mundo en los términos en los que el mundo actual es distinto del de primeros del siglo xx y mucho más del existente en el Siglo i. Lo tecnológico es parte fundamental de esos nuevos mundos y alguna tecnología básica está en el centro de los grandes cambios que los crean, así como las nuevas concepciones científicas e intelectuales generadas por los nuevos conocimientos y nuevas tecnologías ${ }^{7}$.

Las siete cosmovisiones a las que me refiero son: 1) la animista de la antigüedad; 2) la intelectual, de la lógica y del pensamiento de la Grecia Clásica; 3) la cristiana de la Edad Media europea; 4) la traída consigo por El Renacimiento, la Revolución Científica, la Revolución Industrial y la Ilustración de los siglos XV a XVIII en Europa; 5) la creada por el segundo principio de la termodinámica y el concepto de entropía, el descubrimiento del electromagnetismo y la aparición del atomismo del siglo xIx; 6) la aportada por las teorías mencionadas de la Relatividad y de la Mecánica Cuántica; y 7) la cosmovisión en la que estamos hoy causada en mi opinión por el impacto de la anterior y por la difusión entre los hombres de los conceptos relacionados con la llamada «Convergencia NBIC $»^{8}$.

Con referencia a la Relatividad y a la Mecánica Cuántica, y con más énfasis en la segunda, las dos están todavía en fase de ser conocidas y entendidas por la generalidad de las personas y a punto, por tanto, de afectar al conjunto de la sociedad a través de aplicaciones más prácticas y diarias de las ya conseguidas.

\footnotetext{
5 BBVA, c@mbio. cómo Internet está cambiando nuestras vidas, Madrid, 2014.

6 Martin Hilbert y Priscila López, «The World's Technological Capacity to Store» Science,332. numero $6025,2011,60-65$

7 Adolfo Castilla, «Prospectiva de las ideas», Capítulo del libro, Prospectiva e Innovación, editado por Enric Bas, Capítulo Español del Club de Roma, Madrid, 2012

8 Ver blog del autor, www.tendencias21.net/cientecno
} 


\section{LA SÉPTIMA COSMOVISIÓN}

En cuanto a la Convergencia NBIC, habría que hacer referencia dentro de ella a los avances recientes de la humanidad en los terrenos de la biología; de la nanociencia, o conocimiento profundo del interior de la materia; de las ciencias cognitivas, o conocimiento del cerebro; y de la información en un sentido amplio. La nueva cosmovisión estará causada por las nuevas áreas tecnológicas en fase de evolución de la biotecnología, la nanotecnología y la cognotecnología. Áreas de actividad que junto con las llamadas infotecnologías han dado lugar a lo que se conoce, indico de nuevo, como «Convergencia NBIC (Nano-Bio-Info-Cogno)».

Dos importantes informes publicados en la pasada década proporcionaron el nuevo marco de reflexión sobre lo que es nuestro mundo y lo que hacemos los hombres en él.

El primero fue realizado por la NSF (National Science Foundation) en 2002 y lleva el título de "Converging Technologies for improving human performance».

El segundo realizado por la Comisión Europea en 2004 lleva por título, "Converging Technologies- Shaping the Future of European Societies»

Los dos, pero especialmente el primero, creen que estamos a las puertas de una mejora profunda del hombre mismo, de su fisiología y de su inteligencia.

La década de 1990 fue declarada por los Estados Unidos como la del cerebro, y la actual, según algunos autores, debe ser considerada como la década de la consciencia. La Inteligencia Artificial y, sobre todo, la Inteligencia Artificial Fuerte son dos de las grandes áreas de investigación actuales ${ }^{9}$.

\section{Inteligencia Artificial en la Red}

Para añadir algo sobre lo que puede ocurrir en términos de Inteligencia Artificial comienzo indicando que desde hace años vengo elaborando un blog denominado Cognotecnología (www.tendencias21.net/cogno) en el que trato, entre otras muchas cosas, de los avances de la «Inteligencia Artificial Fuerte» y de las máquinas espirituales. Me he referido en él a muchos de los trabajos y publicaciones de Ray Kurzweil y de científicos mucho más avezados sobre el tema de la Consciencia como Christof $\mathrm{Koch}^{10}$, alumno y colaborador de Francis Crick, Giulio Tononi ${ }^{11}$ y varios otros.

Las ideas de estos dos últimos autores y el concepto de "Información Integrada» aportada por el segundo de ellos, aplicadas a la inteligencia de las máquinas, a su crecimiento exponencial y a la interconexión de muchas de ellas a través de Internet, lleva a cálculos realmente notables. Sólo hay que pensar, según Koch, en los billones de ordenadores actuales existentes en el mundo y en los cientos de millones de transistores que los constituyen y podrían quedar integrados masivamente a través de Internet. Koch calcula que sólo en la actualidad se podría hablar de más de 10 elevado a 18 transistores interconectados y compara ese número - asumiendo además que un transistor sea equivalente a una neurona y sus sinapsis- con el número 10 elevado a 15 de sinapsis de un cerebro humano. En una palabra, la complejificación extrema de la que probablemente ha surgido

\footnotetext{
9 Emilio Fontela y Adolfo Castilla, Ingeniería Española 2003. Instituto de la Ingeniería de España. Madrid 2003.

10 Christof Koch,, La Consciencia. Una aproximación neurobiológica, Ariel, Barcelona.

11 Giulio Tononi, Tononi, PHI. A Voyage from the Brain to the Soul, Pantheom Books, New York.
} 
el cerebro y la mente de los seres sentientes, primero, y conscientes, después, está ya en nuestras máquinas. Está en Internet, la cual puede llegar a ser algo equivalente a la Noosfera a la que se refirió el jesuita francés Pierre Teilhard de Chardin (1881-1955).

Me permito en este sentido hacer una consideración personal en relación con esa posibilidad de que Internet termine transformándose en una especie de inteligencia para nuestro planeta. Se trata de que en mi opinión es mucho más probable que lo que llamamos una «máquina espiritual» (es decir, una máquina consciente), se produzca a través de, o en, Internet, que a través de, o en, los ordenadores aislados. Los ordenadores necesitan mucho todavía para llegar a ser verdaderamente inteligentes, o espirituales. Necesitan ser indeterminados e inciertos (lo que podría conseguirse quizás si se construyeran ordenadores cuánticos) pero, sobre todo, deberían estar dotados de «impulsos vitales», es decir, de algo incluido en el interior de sus circuitos y de sus transistores que los hicieran ser capaces de crear por sí mismos conexiones, capaces de cambiarlas automáticamente, y, capaces, por fin, de hacer desparecer aquellas que no sean necesarias en un momento determinado. La plasticidad del cerebro humano será siempre muy difícil de reproducir en una máquina.

\section{INTERNET COMO NOOSFERA}

Da la impresión de que la verdadera inteligencia será más fácil que aparezca en Internet que en un ordenador aislado y que será la humanidad interconectada a través de la Red la que tendrá la posibilidad de dar lugar a una nueva consciencia, una consciencia planetaria en este caso. Para ello será necesario que los hombres utilicemos Internet para actividades más serias y profundas de lo que hacemos hoy, es decir, que lo hagamos para actividades cognitivas, intelectuales, culturales y artísticas, en vez de para simples tareas de intercambio de información convencional, entretenimiento, diversión o relaciones superficiales.

Internet debe dar lugar a través de la interconexión de máquinas y cerebros humanos a un nuevo nivel de ideas abstractas. Unas ideas más elevadas, más comprensivas y más perfectas en todos los sentidos que las ideas de menor nivel producidas por los actuales cerebros humanos. Unas ideas (y unos consensos) que sirvan para encontrar las soluciones a los problemas de nuestro mundo que no acaban de encontrar los hombres relativamente aislados de hoy. Dichos «hombres aislados» y sus ordenadores deben cargarse de información y de inteligencia y su integración a través de redes sociales diversas y a través de foros de debate virtuales debe producir la nueva mente planetaria y las nuevas ideas abstractas de una forma superior de vida. Dichas ideas consensuadas, elaboradas y de más nivel que las de los individuos aislados, volverán a ellos, y por lo tanto el hombre no será una simple «neurona» del nuevo sistema, sino un ser enriquecido y completado.

La idea de esa Internet inteligente, y quizás consciente, se acerca mucho, no tenemos que insistir en ello, a la idea de Noosfera de Teilhard, aunque, desde luego, no es exactamente lo mismo. Se podría decir que Internet adecuadamente utilizada facilitaría la aparición en nuestro mundo de una verdadera Noosfera.

7. IDEAS SOBRE EL MUNDO AL QUe DARÁ LUGAR LA PRESENTE COSMOVISIÓN.

Las consideraciones hechas hasta ahora, especialmente las relacionadas con la Inteligencia de la Red, son componentes de la nueva cosmovisión que se gesta entre 
nosotros, y la cuestión, tras lo indicado, es indicar algo sobre el mundo al que dará lugar tal cosmovisión. Para ello conviene recordar lo ocurrido en el caso de la cuarta cosmovisión, una de las más claras en cuanto a nuevas concepciones e interpretaciones de nuestro mundo y en cuanto a creación de un mundo tecnológico, económico y social radicalmente distinto al previamente existente, deducido de ellas. Dicha gran cosmovisión surge, como ya he dicho, en Europa a través de los siglos xV a XvIII y da lugar al mundo de la construcción de máquinas y bienes industriales y al fuerte desarrollo de la ciencia y la tecnología que nos ha llevado a lo que somos hoy.

Algunos elementos de esa cuarta cosmovisión tienen que ver con: 1) el grito de liberación personal que supuso el Renacimiento, con sus búsqueda del hombre y su énfasis en el humanismo; 2) el interés por la naturaleza y sus leyes y por la construcción de la ciencia moderna; 3) el mecanicismo deducido de la interpretación mecánica del mundo; 4) la interpretación puramente material del hombre mismo, lo cual llevó a considerarlo por algunos como una simple máquina; 5) la secularización de la sociedad y el abandono paulatino de la idea de Dios; 6) la negación de la existencia de poderes públicos absolutos (reyes y otros); 7) la difusión de las ideas de igualdad, derechos humanos y democracia; 8) la orientación de la mente humana y de la acción de los hombres hacia el conocimiento de la naturaleza, hacia la construcción de máquinas e infraestructuras y hacia la transformación y explotación del planeta; y 9) la creación del capitalismo y de la economía moderna basada en el consumo y la inversión.

Son características listadas a vuelapluma que intentan establecer una relación entre las nuevas concepciones sobre nuestro mundo y sobre lo que el hombre hace en él (la esencia de una cosmovisión según mi propia interpretación) con lo que terminamos haciendo, creando y construyendo en términos tecnológicos, económicos y sociales. Asunto al que se han referido muy bien en tiempos recientes autores como Richard Tarnas en "La pasión de la mente occidental»" de la humanidad» ${ }^{13}$, y muchos otros.

El mundo y su evolución pueden interpretarse como un continuo producto de las cuatro a capacidades básicas del hombre a las que tantas referencias vengo haciendo en este trabajo, pero parece más razonable creer que periódicamente se aportan nuevas y radicales interpretaciones que hacen a los hombres cambiar sus concepciones y, especialmente, sus actuaciones. ¿Qué otra cosa podemos pensar, por ejemplo, ante el hecho de que un personaje notable como Eintein, en un momento preciso, aporte una visión del mundo radicalmente distinta de la previamente existente?. ¿O, de que más adelante en el siglo xx, otros personajes como Bohr y Heisemberg contribuyeran a formular una teoría como la Mecánica Cuántica relativa al funcionamiento del interior profundo de la materia que no sólo nos impresiona sino que nos sobrecoge?.

Ideas por cierto que constituyen el fundamento de la sexta cosmovisión, según mis cuentas, y que con las nuevas contribuciones de la NBIC (Nano-Bio-Info-Cogno), en parte deducidas de las mismas Relatividad y Mecánica Cuántica, están dando lugar a la séptima.

¿Qué mundo cabe esperar de estas nuevas concepciones?. Esa es una de las preguntas que muchos nos hacemos. Para dar una contestación a ella sería muy importante listar los componentes de la nueva cosmovisión, a los que ya se ha hecho referencia en parte en los dos últimos apartados.

12 Richard Tarnas, La pasión de la mente occidental, Atalanta, ATLANTA, Girona, 2008

13 Peter Watson, Ideas. Historia intelectual de la humanidad, CRÍTICA, Barcelona, 2005 
Además de ser la época de la profundización del conocimiento sobre el interior de la materia, el interior de la fisiología humana, y, muy especialmente, el interior del cerebro, es también la época de un renovado interés por la mente, el espíritu y la consciencia. Una época hacia la que vamos en la que el hombre actuará sobre su propia naturaleza biológica, sobre su cerebro y su mente, para mejorarlos, en la que probablemente conseguirá aumentar su esperanza de vida y en la que es posible que termine creando inteligencia y consciencia en meras máquinas, además de conseguir, iquién sabe!, que se reproduzcan como lo hacemos nosotros.

El transhumanismo y el hombre posthumano son dos posibilidades que se manejan hoy, y cada vez con más seriedad, así como la aparición de «ciborgs» o nuevos seres mezcla de hombre y máquina. Los robots de tipo humanoide puede que sean unos de los grandes productos de esta época en términos de tecnología y máquinas.

De todo ello, a pesar de la amenazadora tecnología que se vislumbra, lo más destacable puede ser la evolución del espíritu y la consciencia y su potenciación frente el empuje vigoroso de la ciencia. Es posible en este sentido que consigamos, no una ciencia de la consciencia, de la que hablan algunos hoy, sino de una manera nueva de enfrentarnos a los temas del espíritu y de la mente más consistente que la actual y que permita tratar de tú a tú a los avances de la ciencia.

\section{Mecánica Cuántica y Universo Consciente}

La Mecánica Cuántica da pie a lo anterior, sobre todo de la mano de autores como Henry P. Stapp (1928-), físico especializado en mecánica cuántica, actualmente en el «Lawrence Berkeley National Laboratory» de California, que es el autor de uno de los capítulos del destacado libro "Information and the Nature of Reality» ${ }^{14}$, y del libro "Mindfull Universe. Quantum Mechanics and the Participating Observer» ${ }^{15}$.

En ese último libro expone sus ideas en cuanto a que el famoso "colapso de la función de onda» (es decir, la aparición de un electrón, por ejemplo, como partícula, o la transformación de una onda en partícula, al observarla o al medir dónde se encuentra y cuál es su momento), se produce sólo cuando la consciencia interactúa con las ondas (sigue en este aspecto la interpretación más ortodoxa de la mecánica cuántica tal como la formularon Bohr y Heisenberg). Indica en concreto, que la partícula surge cuando una mente consciente selecciona una de las alternativas existentes en las posibilidades cuánticas (es decir, en el «estado cuántico» de un sistema físico en el ámbito de la mecánica cuántica). Como aclaración conviene recordar que en la mecánica cuántica (es decir la mecánica de los sistemas físicos a nivel cuántico o subatómico) los sistemas físicos vienen representados por un «vector de estado» para estados puros y por una matriz de densidad para estados mixtos. Ambos permiten predecir probabilísticamente los resultados de la medición de un observable físico.

Dicho libro, no sólo es uno de los mejores sobre mecánica cuántica que yo he leído, sino que está lleno de nuevas ideas y de interpretaciones muy novedosas, además de revisar con gran claridad y de forma breve, en la mayoría de los casos, las aportaciones de científicos destacados en el terreno de las relaciones mente-cerebro y consciencia-materia

14 Paul Davies y Niels Henrik Gregersen (Editores), Information and the Nature of Reality, From Physics to Metaphysiscs, Cambridge University Press, Cambridge, 2010

15 Henry P, Stapp, Mindful Universe. Quatum Mechanics and the Participating Observer, Springer, Berlin, 2011 
como, John von Neumann (1903-1957), Eugene Wigner (1902-1995) , Michael S. Gazzaniga (1939-), Roger Penrose (1931-) y varios otros. Así como, claro está, de analizar de forma muy profunda la obra de los propios creadores de la Teoría Cuántica, Niels Bohr (1885-1962), Werner Heisenberg (1901-1976 y Wolfgang Pauli (1900-1958).

Su afirmación general más destacada es que el dualismo (existencia de dos naturalezas en nuestro mundo, la física, o material, y la espiritual, o inmaterial), en lo que creyó Platón (427-347 a. C), en la que han creído muchos otros filósofos, y que reformuló Descartes (1596-1650) en el siglo XVII, está de vuelta en nuestro mundo. Muchos científicos a lo largo de los tres últimos siglos lo habían hecho desaparecer en favor del monismo, es decir, la existencia de una sola naturaleza en nuestro mundo: la física o material.

Hay que decir que el dualismo puro o tajante que habla de dos naturalezas distintas y separadas no es admitido hoy por muchos de los que creen fuertemente en el espíritu y la consciencia, ya que o bien creen que hay un substrato más profundo en el que los dos están unidos o que sería mejor hablar de un «monismo trascendente»

Yo me adscribo a esa posición pero hay que reconocer que esa vuelta al dualismo que Stapp defiende, aunque sea en términos relativos, es resultado de todo lo que se va conociendo sobre el interior microscópico del átomo y de la teoría cuántica que explica el funcionamiento de las partículas subatómicas, es decir, el interior profundo de la materia. En dicho funcionamiento, o mejor dicho, en la formación de la realidad física de la que dependemos, interviene la consciencia humana de forma expresa y directa.

La consciencia, la información, el conocimiento, el significado y el propósito, existen en nuestro mundo antes que la materia e independientemente de ella. Nuestro universo es algo consciente en sí mismo, como también parecen querernos decir la cosmología moderna y cosmólogos destacados como Nancy Ellen Abrams y Joel R. Primack ${ }^{16}$.

Stapp explica en su libro los motivos por los que la interpretación dualista del mundo había desaparecido generalizadamente de nuestras concepciones hasta ahora.

El materialismo que con tanta fuerza defendieron y difundieron los ilustrados y enciclopedistas, comenzando por Diderot, que consideraban al hombre como una mera máquina, y el poder que la ciencia ha adquirido en nuestro mundo, han sido responsables de ello. La ciencia moderna, por otra parte, ligada a la experimentación y surgida, como sabemos, alrededor de las ideas de Isaac Newton y los «gigantes» en cuyos hombros se apoyó, describían un mundo muy determinista y una materia, como tantas veces se ha dicho, "sólida, masiva, dura e impenetrable». Todo en la física de Newton tenía una causa cercana y local y todo se producía a través del contacto físico, aunque, por decir toda la verdad, su principal contribución en cuanto a la gravedad, se refería a la actuación a distancia de unos cuerpos sobre otros. Algo de naturaleza desconocida hasta que en 1919 Einstein formulara su Teoría General de la Relatividad.

Stapp dice que nos hemos acostumbrado a olvidar, e incluso a despreciar, la componente inmaterial de nuestro mundo, y eso parece llevar al reduccionismo de la ciencia y al nihilismo de la sociedad, por un lado, y a la imposibilidad de abrir perspectivas más amplias para el descubrimiento de la verdad, por otro.

Hay motivos en ese sentido para preferir el misterio al nihilismo y desde luego yo me inclino por lo primero.

Pero Stapp y los estudiosos de la Teoría Cuántica van más lejos al insistir en que el objetivo de la ciencia no es conocer lo que hay fuera de nosotros sin más, pensando que es fijo y determinado, sino lo que los hombres podemos conocer (diríamos que

16 Nancy Ellen Abrams y Joel R. Primack, "El Nuevo Universo y el Futuro de la Humanidad. Cómo la nueva ciencia del cosmos transformará el mundo». Antoni Bosch editor, S.A., Barcleona, 2013. 
imaginando) y hacer por conocer más, utilizando nuestros pensamientos, ideas, creencias y valores. La Teoría Cuántica tiene que ver con las acciones de adquisición de conocimientos que los hombres emprendemos y con los conocimientos que adquirimos ejecutando dichas acciones.

La diferencia radical con el universo materialista es que las intenciones basadas en nuestras concepciones y valores pueden actuar sobre nuestro comportamiento, sobre nuestras actuaciones sociales y sobre la realidad física, la cual, esta última, se sostiene o es sostenida, por los procesos de nuestras experiencias conscientes. Menciona el caso de la psicología, en la cual la identidad y la forma de una percepción que entra en un proceso consciente depende fuertemente de la mente que la busca. Una persona tiende a experimentar lo que ella misma está buscando, siempre que tal potencialidad exista. El observador no crea lo que no está potencialmente allí pero extrae de lo existente la potencialidad que le interesa y tiene sentido para él.

Las explicaciones cuánticas muestran similaridades con las de la psicología y llevan a pensar que tanto ésta como la física revelan la influencia del observador en la realidad.

A la mecánica cuántica en general y a sus relaciones con la biología y con el psiquismo ha dedicado mucha atención y publicado profusamente el Padre Jesuita Javier Monserrat, con el que me une una gran amistad y del que continuamente aprendo sobre estos complicados temas. Su libro La percepción visual, una obra enciclopédica donde las haya, es una referencia imprescindible en algunos de temas, tocados ligeramente en este trabajo, en especial en todo lo relacionado con la arquitectura del psiquismo humano y sus relaciones con lo biología y con la mecánica cuántica ${ }^{17}$

Hay artículos diversos de Monserrat sobre estos temas que hemos estudiado también en el proceso de redacción de este trabajo. Entre ellos, «Los paradigmas de la neurociencia actual», "La imagen física del universo y la neurociencia», "Neurociencias: identidad personal y pervivencia humana». Asimismo hemos utilizado trabajos del joven pero destacado investigador, Manuel Béjar Gallego, del que hemos analizado entre otros trabajos, "Neurología Cuántica» ${ }^{18}$.

\section{Conclusiones}

Como final de unas reflexiones ligeras y superficiales, aunque bienintencionadas en cuanto a ser personales, de las que no me siento muy satisfecho, por supuesto, debo decir que he tratado de sacar partido de una forma de ver la evolución del hombre basada en lo que he llamado «cosmovisiones». Las he definido como nuevas interpretaciones sobre nuestro mundo y sobre lo que los hombres hacemos en él producto de grandes descubrimientos y grandes ideas aportadas por determinados personajes notables que captan en poco tiempo la atención de todos. Se mezclan en ellas no sólo las ideas abstractas del hombre sino sus descubrimientos científicos, la tecnología que basada en ellos desarrolla, el arte que genera y la economía y sociedad que termina creando con las nuevas concepciones sobre nuestro mundo como base y fundamento.

He sugerido la ocurrencia en la historia de la humanidad de siete grandes cosmovisiones y me he detenido en explicar lo que podría ser la séptima en la que actualmente

17 Javier Monserrat, La percepción visual. La arquitectura del psiquismo desde el enfoque de la percepción visual. Segunda edición revisada y ampliada. Biblioteca Nueva, Madrid, 2008

18 Una gran parte de estos artículos han sido publicados en Pensamiento. Revista de Investigación e Información Filosófica. Universidad Pontificia Comillas 
estamos entrando. Tiene que ver, si no me equivoco, con el impacto entre nosotros de interpretaciones ya antiguas como la Teoría de la Relatividad y la Mecánica Cuántica, combinadas con avances recientes como los producidos alrededor de la Convergencia NBIC.

He tratado de mostrar algunas características de la nueva cosmovisión, incluyendo el renovado interés por la mente, la consciencia y el espíritu, la creciente aceleración del cambio tecnológico, los anunciados avances en la posible mejora del hombre fisiológica y mentalmente, la evolución de la Inteligencia Artificial Fuerte y la creación de humanoides y la aparición de fenómenos tan deslumbrantes como el hombre posthumano.

La Mecánica Cuántica en particular ha sido identificada como fundamento de gran parte de lo puede ocurrir, especialmente en cuanto al nuevo poder de la mente y del espíritu, en lo que especialmente me he detenido.

He concluido con una atención algo más especial a este último tema utilizando para ello la obra de un autor como Henry P. Stapp que claramente apuesta por un universo consciente y por un poder del espíritu sobre la materia superior al que la ciencia actual pretende.

\section{Bibliografía}

Abrams, N. E. y Primack, J. R. (2013), «El Nuevo Universo y el Futuro de la Humanidad. Cómo la nueva ciencia del cosmos transformará el mundo». Antoni Bosch editor, S.A., Barcleona.

Altman, D. (2011), Futuros Imperfectos. Las 12 tendencias asombrosas que remodelarán la economía global, Tendencias Editores, Barcelona.

Bartra, R. (2014), Antropología del Cerebro. Ciencia, cultura y libre albedrio, Pre-Textos, Valencia.

Blumenberg, H. (2013), Historia del espíritu de la técnica, Pre-Textos, Valencia.

Brynjolfsson, E. y McAfee, A. (2013), La carrera contra la máquina, Antoni Bosch Editor, S. A., Barcelona.

Buzan, B. y Segal, G. (1999), El futuro que viene, Editorial Andrés Bello, Barcelona.

Castells, M. (2003), The Information Age: Economy, Society and Culture, Blackwell, Oxford.

- (2001), The Internet Galaxy. Reflections on the Internet Business and Society, Areté, Barcelona.

Davies, P. y Gregersen, N. H. (Editores) (2010), Information and the Nature of Reality, From Physics to Metaphysiscs, Cambridge University Press, Cambridge.

Deacon, T. W. (2013), Naturaleza Incompleta. Cómo la mente emergió de la materia, Tusquets Editores, Barcelona.

De Grey, A. y RAE, M. (2013), El fin del envejecimiento, lola books. Berlín.

Diamandis, P. H. y KotLer, S. (2013), Abundancia. El futuro es mejor de lo que piensas, Antoni Bosch Editores, Barcelona

Dyson, F. (1998), Mundos del futuro, Crítica, Barcelona.

Franklin, D. y ANDrews, J. (Coordinadores) (2013), El mundo en 2050. Todas las tendencias que cambiarán el planeta. The Economist, Grupo Planeta, Barcelona.

Gleick, J. (2012), La Información. Historia y Realidad, Crítica, Barcelona.

Gore, A. (2013), The Future. Six drivers of global change, Random House, New York.

HaYles, N. K. (1999), How we Became Posthuman, The University of Chicago Press, Chicago.

Hölscher, L. (2014), El descubrimiento del futuro, Siglo xxi de España, Madrid.

Johnson, S. (2013), Futuro perfecto. Sobre el progreso de la era de las redes, Turner, Madrid.

KAKU, M. (2014), El futuro de nuestra mente, Debate, Barcelona.

Косн, C. (2005), La Consciencia. Una aproximación neurobiológica, Ariel, Barcelona. 
KuRzweIL, R. (2012), La singularidad está cerca. Cuando los humanos transcendamos la biología, lola books, Berlín.

Kurzweil, R. (2013), Cómo crear una mente. El secreto del pensamiento humano. Lola books, Berlín. Miller, J. D. (2012), Singularity Rising, Benbella Books, Dallas, TX, Estados Unidos.

Monserrat, J. (2008), La percepción visual. La arquitectura del psiquismo desde el enfoque de la percepción visual, Biblioteca Nueva, Madrid.

Monserrat, J. (2005), Hacia un nuevo mundo. Filosofía Política del protagonismo histórico emergente de la sociedad civil, Publicaciones de la Universidad Pontificia Comillas, Madrid.

Mosterín, J. (2013), Ciencia, filosofía y racionalidad, Gedisa Editorial, Barcelona.

RANDALL, L. (2013), Llamando a las puertas del cielo, Acantilado, Barcelona.

RIFKIN, J. (2011), La tercera revolución industrial, Paidós, Barcelona.

- (2013), La era del acceso. La revolución de la nueva economía, Paidós, Barcelona.

Rosenblum, B. y KutTner, F. (2013), El enigma cuántico. Encuentros entre la física y la conciencia, Tusquets, Barcelona.

Sмiтн, L. C. (2011), El mundo del 2050. Las cuatro fuerzas que determinarán el futuro de la civilización, Debate, Barcelona.

SCHRöDinger, E. (2006), ¿Qué es la vida?, METATEMAS, Tusquets Editores, Barcelona.

StapP, H. P. (2011), Mindful Universe. Quatum Mechanics and the Participating Observer, Springer, Berlin.

SwaAB, D. (2014), Somos nuestro cerebro. Cómo pensamos, sufrimos y amamos, Plataforma Editorial, Barcelona.

TARNAS, R., La pasión de la mente occidental, Atalanta, ATLANTA, Girona, 2008

Tononi, G. (2012), PHI. A Voyage from the Brain to the Soul, Pantheom Books, New York.

Turney, J. (2010), The Rough Guide to The Future, Rough Guides, Penguin Group, Victoria, Australia.

Van SANTEn, R., Khoe, D. y Vermeer, B. (2010), 2030. Technology that will change the World, Oxford University Press, Oxford.

Watson, P. (2000), Ideas. Historia intelectual de la humanidad, CRÍTICA, Barcelona.

Universidad Antonio de Nebrija, Madrid

Adolfo Castilla

acastillag@telefonica.net

[Artículo aprobado para publicación en diciembre de 2014]. 